\title{
Interplay Effect of Excitation and Temperature on Carrier Transfer between Vertically Aligned InAs/GaAs Quantum Dot Pairs
}

\author{
Yao Liu ${ }^{1}$, Ying Wang ${ }^{1}$, Baolai Liang ${ }^{1, *}$, Qinglin Guo ${ }^{1}$, Shufang Wang ${ }^{1}$, Guangsheng Fu ${ }^{1}$, \\ Yuriy I. Mazur ${ }^{2}$, Morgan E. Ware ${ }^{3}$ and Gregory J. Salamo ${ }^{2}$ \\ 1 College of Physics Science \& Technology, Hebei University, Baoding 071002, China; \\ m15100299616_2@163.com (Y.L.); hbuwangying@126.com (Y.W.); qlguo@hbu.edu.cn (Q.G.); \\ sfwang_researcher@aliyun.com (S.W.); fugs@hbu.edu.cn (G.F.) \\ 2 Institute for Nanoscience and Engineering, University of Arkansas, Fayetteville, AR 72701, USA; \\ ymazur@uark.edu (Y.I.M.); salamo@uark.edu (G.J.S.) \\ 3 Department of Electrical Engineering, University of Arkansas, Fayetteville, AR 72701, USA; \\ meware@uark.edu \\ * Correspondence: liangbaolai@gmail.com; Tel.: +86-312-507-9354
}

Academic Editor: Paul J. Simmonds

Received: 1 September 2016; Accepted: 8 November 2016; Published: 10 November 2016

\begin{abstract}
Carrier transfer in vertically-coupled InAs/GaAs quantum dot (QD) pairs is investigated. Photoluminescence (PL) and PL excitation spectra measured at low temperature indicate that the PL peak intensity ratio between the emission from the two sets of QDs-i.e., the relative population of carriers between the two layers of QDs—changes with increasing excitation intensity. Temperature-dependent PL reveals unexpected non-monotonic variations in the peak wavelength and linewidth of the seed layer of QDs with temperature. The PL intensity ratio exhibits a "W" behavior with respect to the temperature due to the interplay between temperature and excitation intensity on the inter-layer carrier transfer.
\end{abstract}

Keywords: photoluminescence; self-assembled quantum dots; carrier transfer; semiconductor

\section{Introduction}

Self-assembled InAs/GaAs quantum dots (QDs) have attracted enormous attention over the past two decades due to their unique characteristics and huge potential for device applications [1-5]. In particular, the vertically-stacked InAs/GaAs QDs have been widely applied as a promising structure in many optoelectronic devices, including emitters, infrared detectors, and photovoltaics, as well as devices for optical computations [6-11]. As a good example, coupling two layers of vertically aligned QDs to fabricate artificial QD-molecules not only opens a new way to investigate quantum phenomena over a wide range of configurations, but also provides an approach to implementing quantum entangled states [12-15]. Such bilayer QD structures enable flexible manipulation of the quantum coupling between two layers of QDs, independently controlling the QD density, size, and uniformity. These advantages make the bilayer InAs/GaAs QD structures an interesting choice for QD device applications [16-18].

Despite such significant potential for device applications, it is very important to understand how the QD pairs are coupled, and how their proximity may affect their optical and carrier transfer properties. Yet studies of the energy transfer properties are indispensable when attempting to understand the performance of optoelectronic devices based on bilayer InAs/GaAs QD structures. In our previous work, we have successfully manipulated the quantum coupling between InAs QD pairs by inserting a thin $\mathrm{Al}_{0.5} \mathrm{Ga}_{0.5} \mathrm{As}$ barrier [19]. The effect of the thin $\mathrm{Al}_{0.5} \mathrm{Ga}_{0.5} \mathrm{As}$ barrier on 
the carrier transfer between the dots of a vertically-aligned QD pair has been carefully studied by comparing the optical performance of samples with and without the $\mathrm{Al}_{0.5} \mathrm{Ga}_{0.5} \mathrm{As}$ barrier. In this work, we further enrich the understanding of carrier transfer in such bilayer QDs. The interplay between excitation and temperature on carrier transfer inside the vertically-aligned InAs/GaAs quantum dot pairs is investigated.

\section{Experiments}

The investigated bilayer QD structure consists of two layers of InAs QDs separated by a hybrid $\mathrm{GaAs} / \mathrm{Al}_{0.5} \mathrm{Ga}_{0.5} \mathrm{As} / \mathrm{GaAs}$ spacer. The sample was grown on a semi-insulated $\mathrm{GaAs}(001)$ substrate by solid source molecular beam epitaxy (MBE) with an $\mathrm{As}_{4}$ beam equivalent pressure of $6.0 \times 10^{-6}$ Torr [19]. After a $500 \mathrm{~nm} \mathrm{GaAs}$ buffer layer was grown at $600{ }^{\circ} \mathrm{C}$, the substrate temperature was lowered to $530{ }^{\circ} \mathrm{C}$, and two monolayers (MLs) of InAs were deposited with a growth rate of $0.013 \mathrm{ML} / \mathrm{s}$ to form the seed layer QDs (SQDs). After a $10 \mathrm{~s}$ growth interruption, the hybrid GaAs $/ \mathrm{Al}_{0.5} \mathrm{Ga}_{0.5} \mathrm{As} / \mathrm{GaAs}$ spacer was deposited, consisting of (in order): $10 \mathrm{MLs}$ of GaAs, $15 \mathrm{MLs}$ of $\mathrm{Al}_{0.5} \mathrm{Ga}_{0.5} \mathrm{As}$, and $10 \mathrm{MLs}$ of GaAs. These three layers on the top of the SQDs acted as a wider band gap barrier. Then, the substrate temperature was raised to $600^{\circ} \mathrm{C}$, and the sample was annealed for $8 \mathrm{~min}$. After that, the substrate temperature was lowered to $530^{\circ} \mathrm{C}$ again, and $2.6 \mathrm{ML}$ of InAs were deposited to form the top layer QDs (TQDs). The TQDs were capped by $20 \mathrm{~nm}$ of GaAs at $530^{\circ} \mathrm{C}$, and then an additional $60 \mathrm{~nm}$ of $\mathrm{GaAs}$ at $600^{\circ} \mathrm{C}$.

The cross-sectional Transmission Electron Microscopy (XTEM) and photoluminescence (PL) measurements were implemented after the sample was cooled down and removed from the MBE growth chamber. For PL study, the sample was mounted in a closed-cycle cryostat with temperature variable from $8 \mathrm{~K}$ to $300 \mathrm{~K}$. The sample was excited by a continuous-wave laser operated at $\lambda=532 \mathrm{~nm}$ with excitation intensity between 1 and $10^{6} \mathrm{~mW} / \mathrm{cm}^{2}$. The PL signal was detected by a liquid nitrogen cooled CCD detector array attached to a $50 \mathrm{~cm}$ Acton spectrometer. Time-resolved photoluminescence (TRPL) was measured by a PicoHarp 300 (PicoQuant, Berlin, Germany) time-correlated-single-photon-counting (TCSPC) system with a super-continuum laser as the excitation. For reference, an uncapped SQD sample and an uncapped TQD sample were grown under the same conditions for morphology characterization by atomic force microscope (AFM).

\section{Results and Discussion}

As shown by the AFM images in Figure 1a,b, the uncapped SQDs have an areal density of $5.7 \times 10^{9} \mathrm{~cm}^{-2}$, an average height of $11.7 \mathrm{~nm}$, and an average diameter of $68.5 \mathrm{~nm}$, while the uncapped TQDs have an areal density of $5.5 \times 10^{9} \mathrm{~cm}^{-2}$, an average height of $19.3 \mathrm{~nm}$, and an average diameter of $83.0 \mathrm{~nm}$. No large incoherent islands or defects were observed on the surface, indicating good sample quality for both QD layers. From the AFM images, we found that both layers of QDs had an equal areal density, indicating the possibility that the QDs were vertically aligned into pairs.

The XTEM in Figure 1c confirms that the SQDs and TQDs were vertically correlated into QD pairs through the thin GaAs/AlGaAs/GaAs hybrid-spacer. Such a thin spacer will lead to strong electronic coupling, and hence inter-layer carrier transfer from the small SQDs to the large TQDs. The carrier tunneling for similar bilayer InAs QD structures have been well studied. By using a modified form of the semiclassical Wentzel-Kramers-Brillouin (WKB) approximation, the carrier tunneling time is estimated to depend on the barrier thickness and potential height [19-21]. In addition, as the effective mass of the holes is much larger than the electrons, it is expected that carrier tunneling in the InAs bilayer QD structures is primarily performed by the electrons. This electron tunneling is shown schematically in the band diagram of the bilayer QD structure in Figure 1e.

Vertical carrier transfer is first detected by PL measured at low temperature $(T=8 \mathrm{~K})$, with a laser excitation intensity as low as $3 \mathrm{~mW} / \mathrm{cm}^{2}$ to avoid the appearance of excited QD states. The spectrum in Figure $1 \mathrm{~d}$ shows a discrete double-peak profile. The peak at $E_{\mathrm{T}}=1127 \mathrm{~nm}$ is attributed to the electron-hole ground state transition from the TQDs, while the peak at $E_{S}=1075 \mathrm{~nm}$ is assigned to the 
SQDs. Although the SQDs and TQDs have the same areal density, and therefore should have similar PL intensities, we observed a distinct PL intensity difference caused by carrier transfer from the SQDs to the TQDs. This is driven by the difference in energies of the two QD systems. The high-energy electrons in the ground state of the SQDs tend to tunnel to the lower energy ground state of the TQDs. From PL, we see that this energy difference between $\mathrm{E}_{\mathrm{T}}$ and $\mathrm{E}_{\mathrm{S}}$ is $\sim 53.5 \mathrm{meV}$ (about $52 \mathrm{~nm}$ in PL wavelength), which is likely due to the different QD dimensions. The full-width-at-half-maximum (FWHM) is $17.2 \mathrm{~nm}$ for the TQD peak, but $33.2 \mathrm{~nm}$ for the SQD peak. This indicates that the top layer of QDs have a significantly more uniform size distribution in comparison with the SQDs.
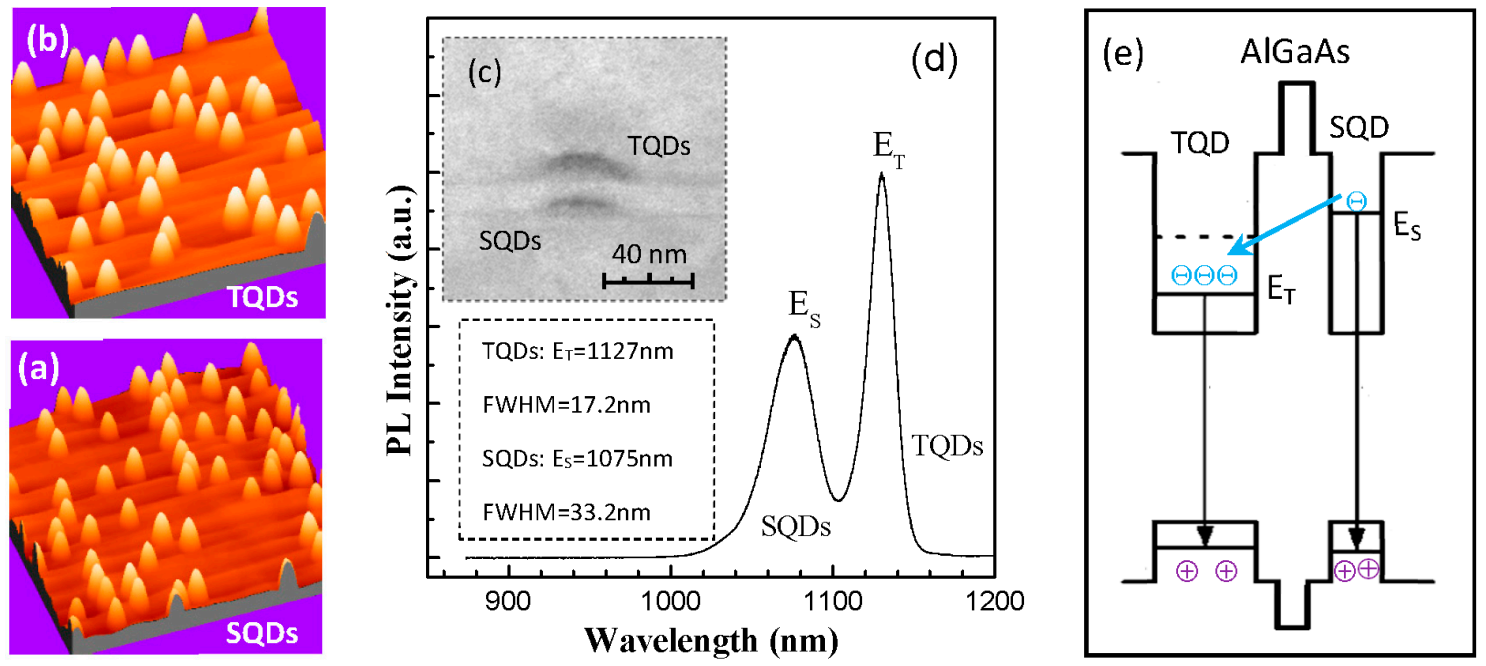

Figure 1. (a) A $1 \mu \mathrm{m} \times 1 \mu \mathrm{m}$ atomic force microscopy (AFM) image of the seed layer quantum dots (SQDs); (b) A $1 \mu \mathrm{m} \times 1 \mu \mathrm{m}$ AFM image of the top layer QDs (TQDs); (c) Cross-sectional transmission electron microscopy (XTEM) image of one QD pair structure; (d) Low temperature $(8 \mathrm{~K})$ photoluminescence (PL) spectrum obtained with a laser excitation intensity of $3 \mathrm{~mW} / \mathrm{cm}^{2}$; (e) The band diagram of the QD pair structure with a $10 \mathrm{~nm} \mathrm{GaAs/AlGaAs/GaAs} \mathrm{hybrid} \mathrm{spacer.}$ FWHM: full-width-at-half-maximum.

Figure 2a plots the PL spectra measured as a function of laser excitation intensity at $T=8 \mathrm{~K}$. Each spectrum is normalized with respect to the maximum TQD peak intensity and shifted up for clarity. When the excitation intensity is above $\sim 3 \times 10^{3} \mathrm{~mW} / \mathrm{cm}^{2}$, one additional PL peak at $\lambda \sim 1095 \mathrm{~nm}$-attributed to the first excited state of TQDs—arises in the PL spectra. Then, as excitation intensity increases further, the excited states of TQDs become more prominent. Finally, the PL signals from the two QD systems fuse together into a very broad feature, where we cannot clearly distinguish the $E_{\mathrm{S}}$ emission from the SQDs. To further investigate the appearance of the excited states, the evolution of the FWHM for the SQDs and TQDs is plotted in Figure 2b. An obvious increase in the FWHM of the SQDs is observed when the laser excitation intensity increases past $\sim 3 \times 10^{3} \mathrm{~mW} / \mathrm{cm}^{2}$, which is very similar to the power at which the first excited state from the TQDs start to influence the SQD spectra, as seen in Figure 2a. Therefore, the excitation intensity was subsequently kept to less than $3 \times 10^{3} \mathrm{~mW} / \mathrm{cm}^{2}$ to avoid the effect of the excited states in the vertical carrier transfer study.

From the PL spectra in Figure 2a, the integrated PL intensities are extracted and plotted in Figure 2c in the low excitation regime to avoid influence from the excited states of QDs. The integrated PL intensity of the ground state emission of InAs QDs can be described by $I_{\mathrm{PL}}=\eta P^{\alpha}$, where $I_{\mathrm{PL}}$ is the integrated PL intensity, $P$ is the laser excitation intensity, and $\eta$ and $\alpha$ are fitting coefficients which define a power law [22,23]. In fitting the experimental data, we find the coefficient $\alpha$ to be approximately equal to unity in both cases, indicating that the main contributing mechanism is the exciton recombination, while carrier losses due to nonradiative recombination are small for both samples. In particular, the slope $\eta$ is closely related to the PL efficiency. We obtain $\eta=0.035$ for the 
TQDs and $\eta=0.023$ for the SQDs. The larger $\eta$ value of the TQDs indicates that they have a better carrier collection efficiency than the SQDs in the low excitation regime, due to carrier transfer from SQDs to TQDs in this vertically-coupled QD pair structure.

It is also evident from Figure 2a that as the laser excitation increases, the SQD peak intensity increases faster than the TQD intensity. In Figure $2 \mathrm{~d}$, the PL peak intensity ratio $(R=\mathrm{SQD} / \mathrm{TQD} \%)$ demonstrates this clearly, as it grows throughout the entire excitation intensity range. Although we suppose that all QDs have the same carrier capture probability from the GaAs matrix, at very low excitation intensity, the large TQDs have an added channel to receive carriers through tunneling from the smaller SQDs. However, as the laser intensity increases, the larger TQDs begin to saturate, turning off the tunneling channel and allowing the small SQDs to keep more carriers, such that the emission from the SQDs increases quickly. It is easy to understand from the observation in Figure $2 \mathrm{~d}$ that the strength of carrier transfer depends on the population of both TQDs and SQDs. There must be both carriers in the SQDs to transfer and available states in the TQDs to populate through the transfer.
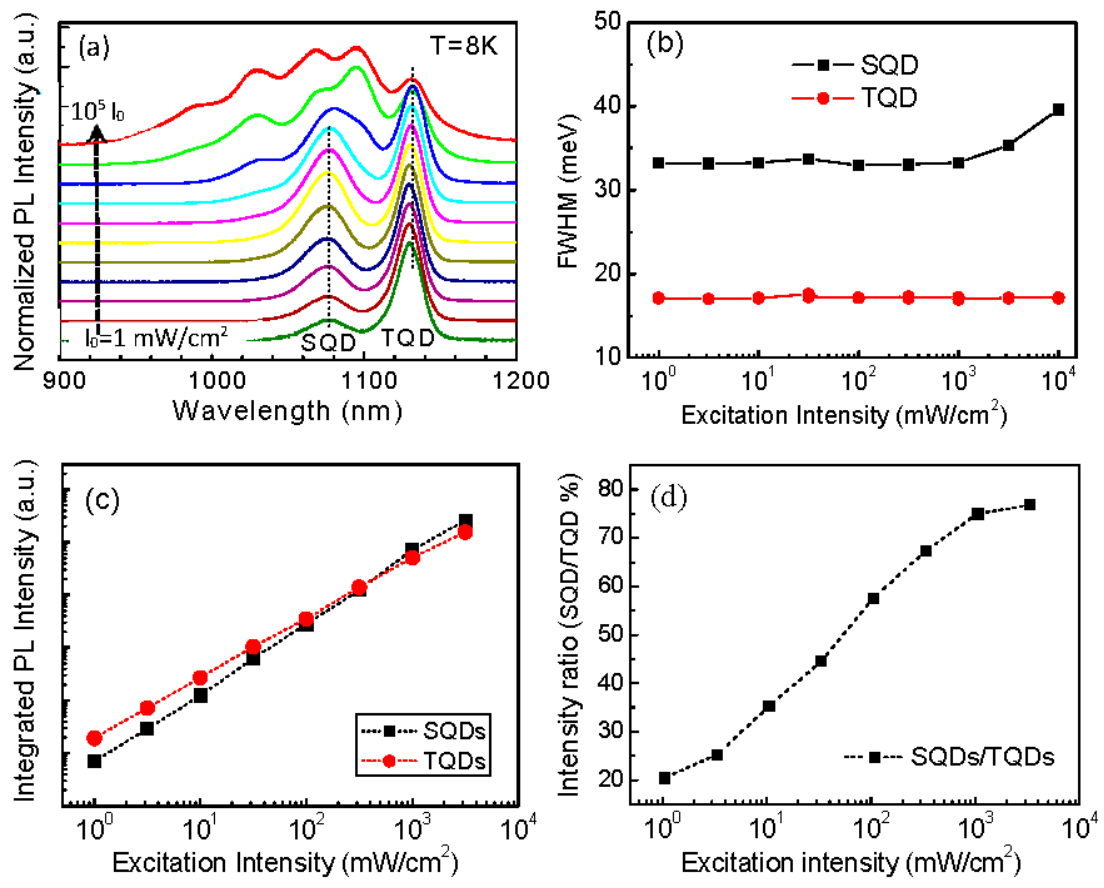

Figure 2. (a) Normalized PL spectra measured at low temperature $(T=8 \mathrm{~K})$ as a function of the laser excitation intensity; (b) Linewidth (FWHM) of the TQDs and SQDs PL band; (c) Integrated PL intensity as a function of the laser excitation intensity; (d) PL intensity ratio of the SQDs and TQDs ( $R=$ SQDs $/$ TQDs $\%)$ as a function of the laser excitation intensity.

For a broader characterization of the carrier transfer between SQDs and TQDs, PL excitation (PLE) spectra are measured by using a Super-Continuum laser with a constant output power of $0.1 \mathrm{~mW}$ at each wavelength. As shown in Figure 3a, the detection is set at the TQD peak wavelength. The PLE spectrum reveals a clear excitation resonance at $820 \mathrm{~nm}$, which is assigned to bulk GaAs, and also resonances at $852 \mathrm{~nm}$ and $918 \mathrm{~nm}$, assigned to the heavy hole and light hole absorption of the wetting layer, respectively. Finally, the PLE spectrum shows a pronounced excitation resonance at the SQD peak $(\sim 1065 \mathrm{~nm})$. As the first excited state of the TQDs overlaps with the ground state of the SQDs, the observed excitation resonance at $\sim 1065 \mathrm{~nm}$ in the PLE spectrum could be a combined contribution of carriers from the first excited state of TQDs and from the ground state of SQDs to the TQD ground states. However, the observed excitation resonance in Figure $3 \mathrm{a}$ is much stronger than similar results reported from PLE measurements of single layers of QDs $[18,24,25]$. This leads to the conclusion that there is a significant transfer of carriers from the SQDs to the TQDs in our QD pair structure [19]. 
We further investigate this carrier transfer by measuring PL spectra with different excitation laser wavelengths and intensities. Figure $3 b$ shows that - independent of excitation wavelength-the PL peak intensity ratio, $R$, increases as the excitation intensity increases. For example, the inset shows the spectra measured with a laser wavelength of $819 \mathrm{~nm}$ and laser excitation intensities of $3 \mathrm{~W} / \mathrm{cm}^{2}$, $0.3 \mathrm{~W} / \mathrm{cm}^{2}$, and $0.03 \mathrm{~W} / \mathrm{cm}^{2}$. Here, the spectra are normalized to the TQD peak intensity, and it is clear that the SQD is growing in relative intensity. This demonstrates that the TQD ground states responsible for this emission are saturating as a result of increasing the excitation intensity. In other words, the TQD ground states available for carriers to transfer into decrease with increasing excitation intensity. Thus, the SQD peak along with the ratio, $R$, increase as the TQD peak saturates. This is apparently true for all excitation wavelengths studied. In addition, there is an enhancement of this ratio, $R$, for excitation around $\lambda=750 \mathrm{~nm}$. This is most likely due to the enhanced absorption of the excitation laser as its wavelength approaches the GaAs band edge. The enhanced absorption generates more carriers inside the GaAs barrier, which consequently saturates the TQD emission faster. However, $R$ remains nearly constant throughout the wetting layer (excitation laser wavelength $\lambda=860 \mathrm{~nm}$ ) and sub-wetting layer (excitation laser wavelength $\lambda=875 \mathrm{~nm}$ ) regions. Therefore, the change in $R$ reflects the carrier transfer from the SQDs to the TQDs, and the dependence on the laser wavelength and the laser power (i.e., the carrier injection level).
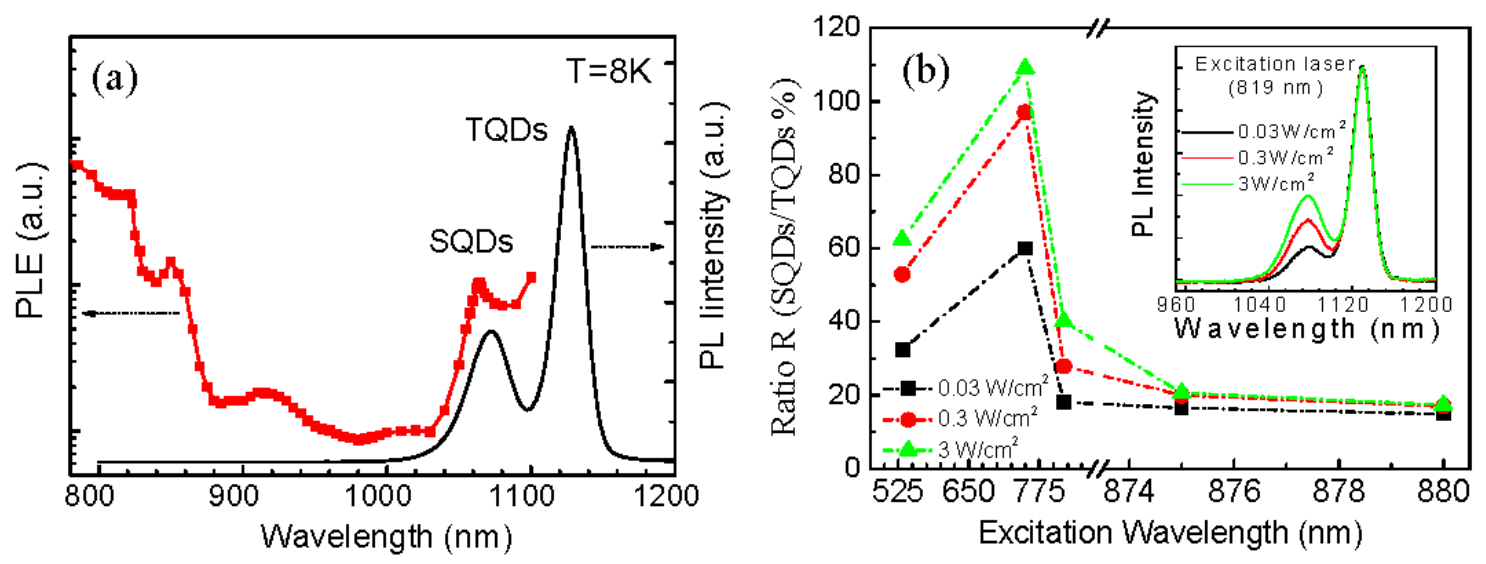

Figure 3. (a) One PL spectrum and one PL excitation (PLE) spectrum with detection at the TQD maximum measured at the low temperature of $8 \mathrm{~K}$. The excitation laser was optimized for PLE measurement at the SQD PL resonance wavelength; (b) The PL peak intensity ratio $R$ (SQD/TQD100\%) as a function of the excitation laser wavelength. Note that the trendlines between data points are added as guides to the eyes only. The inset shows the low temperature PL spectra measured with a laser wavelength of $\lambda=819 \mathrm{~nm}$ at different excitation intensity.

Figure $4 \mathrm{a}$,b shows the temperature-dependent PL spectra from $8 \mathrm{~K}$ to $300 \mathrm{~K}$, with laser excitation intensities fixed at $1000 \mathrm{~mW} / \mathrm{cm}^{2}$ and $100 \mathrm{~mW} / \mathrm{cm}^{2}$, respectively. For convenience, the PL spectrum at each temperature is normalized to the TQD maxima. The PL peak wavelength, FWHM, integrated intensity, and intensity ratio $R$ are extracted and plotted as functions of temperature in Figure 4c-f, respectively, and will be discussed separately below.

Figure 4c shows that both SQD and TQD PL peaks shift $(\sim 90 \mathrm{~nm})$ towards longer wavelengths by increasing the temperature from $8 \mathrm{~K}$ to $300 \mathrm{~K}$ for both high and low power excitation. For the TQDs, the peak wavelength smoothly shifts towards the red as a result of the good QD homogeneity localizing the carriers inside the QDs. The red shift of the TQDs closely follows the well-known Varshni's law, $E(T)=E_{0}-\left(\alpha T^{2}\right) /(T+\beta)$, where $E_{0}$ is the energy gap at $T=0 \mathrm{~K}$, and the fitting parameters are $\alpha=4.58 \times 10^{-4} \mathrm{eV} / \mathrm{K}$ and $\beta=243 \mathrm{~K}[23,26,27]$. However, the temperature dependence of the SQD peak exhibits a rapid red-shift in the temperature range from $\sim 100 \mathrm{~K}$ to $\sim 200 \mathrm{~K}$ for both high and low excitation powers. It is likely that this rapid red-shift is caused by the thermal activation 
of carriers from the SQDs and carrier transfer to the TQDs at increased temperature. Meanwhile, the starting temperature and shift-rates of this rapid red-shift are different at high and low excitation powers, demonstrating that the degree of temperature-induced variation of carrier transfer is also excitation power-dependent.
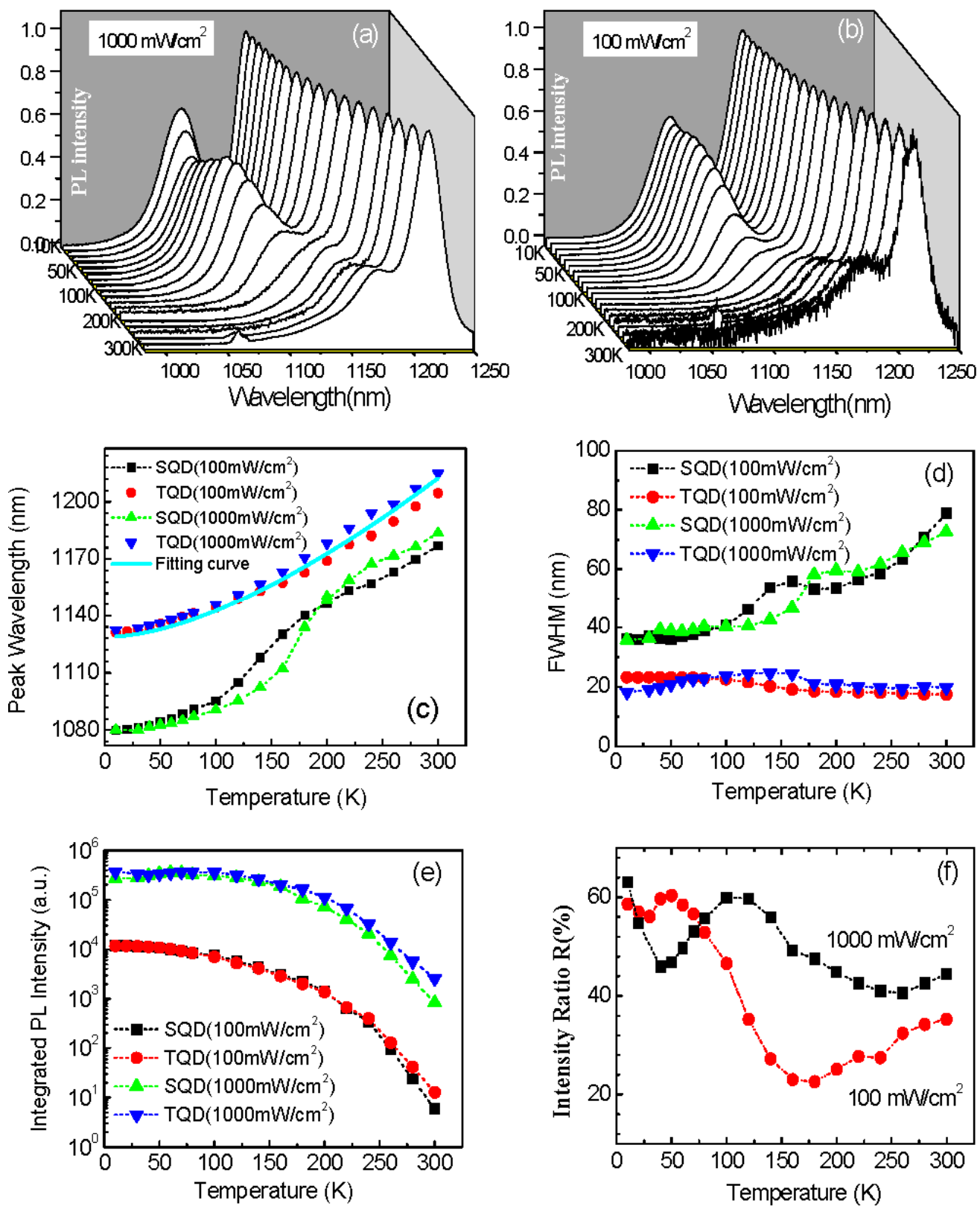

Figure 4. Normalized PL spectra as a function of temperature measured with a laser excitation intensity of (a) $1000 \mathrm{~mW} / \mathrm{cm}^{2}$ and (b) $100 \mathrm{~mW} / \mathrm{cm}^{2}$; (c) The PL peak wavelength; (d) the FWHM; (e) the integrated PL intensity; and (f) the PL intensity ratio as functions of temperature for the SQDs and TQDs, respectively.

Figure $4 \mathrm{~d}$ shows the variation of the FWHM for excitation intensities of both $1000 \mathrm{~mW} / \mathrm{cm}^{2}$ and $100 \mathrm{~mW} / \mathrm{cm}^{2}$. The FWHM of TQDs remains mostly stable through the entire temperature range for both powers, reaffirming the good homogeneity of the TQDs. Similar to the emission energy, though, we find a rapid change in the FWHM through the same temperature range. Here, there is a broadening from $\sim 40 \mathrm{~nm}$ to $\sim 60 \mathrm{~nm}$ for the SQDs for both high and low excitation. However, the onset temperature 
at which the broadening begins is $\sim 50 \mathrm{~K}$ higher for the high power excitation than for the low power. Again, this indicates an interplay between the temperature and excitation intensity.

Figure 4e shows the variation of the integrated PL intensity as a function of temperature for both TQDs and SQDs under excitation intensities of $1000 \mathrm{~mW} / \mathrm{cm}^{2}$ and $100 \mathrm{~mW} / \mathrm{cm}^{2}$. The integrated PL intensity slowly decreases with temperature through the low temperature range $(<100 \mathrm{~K})$ for both powers, then finally decays rapidly at higher temperatures. Similar to Figure $2 c$, we see that the intensities from both populations of QDs are very similar, which is a direct reflection of their densities being the same. However, the subtle differences between the populations-which can be seen in some of the plots of the FWHMs-are much easier to see in plotting the PL intensity ratios, $R$, as functions of temperature, which reflect the relative equilibrium populations between the SQDs and the TQDs. This is plotted in Figure 4f. A special "W" behavior is observed for both excitation intensities. Taking the higher power excitation, $1000 \mathrm{~mW} / \mathrm{cm}^{2}$, as an example, when $<30 \mathrm{~K}$, the PL intensity ratio, $R$, decreases with increasing temperature. After that, $R$ increases from $30 \mathrm{~K}$ to $120 \mathrm{~K}$, then slowly decreases again until $T=260 \mathrm{~K}$, and finally grows slightly to room temperature. Again, these features appear higher in temperature for the high power excitation than for the low power. These " $\mathrm{W}$ " behaviors of the PL intensity ratio show that the population of carriers inside the QDs is strongly dependent on both the temperature and the excitation powers.

In summary of the observations in Figure 4, it can be seen that the peak wavelength, as well as the FWHM of SQDs and the PL peak intensity and the intensity ratio, $R$, have distinct variations in the middle temperature range $(100 \mathrm{~K}-200 \mathrm{~K})$. The performance of this QD-pair sample at low temperatures $(<100 \mathrm{~K})$ can be explained by carrier thermal activation and redistribution within the same QD layer $[23,28]$. In this process, there is very little loss of carriers from the system; therefore, it generally shows a change in the FWHM, but not the integrated PL intensity. It is clear, however, that this process can change the relative carrier populations, and subsequently the carrier tunneling rate between the QDs of the coupled bilayer QD structure. As a result, we observe that the PL intensity ratio decreases at first and then increases, forming one half of the " $\mathrm{W}$ " behavior in Figure $4 \mathrm{f}$.

The prominent variations in the middle temperature range $(>100 \mathrm{~K})$ can be understood by considering that the PL emission from both the TQDs and the SQDs begins to decrease rapidly due to carrier thermal quenching. In this process, both TQDs and SQDs lose carriers to nonradiative centers due to phonon scattering. It is well known that this carrier thermal quenching is strongly dependent on the QD dimensions or the discrete energy levels in the QDs. We observe that the peak wavelength and the FWHM behave differently for TQDs and SQDs in the middle temperature range. Of course, the relative population between the TQDs and SQDs vary as the temperature increases. As a result, we observe the second half of the "W" behavior in Figure 4f. The "W" behavior indicates that the coupled bilayer QD structure has different thermal quenching properties than a single layer of InAs QDs. The inter-layer carrier transfer from SQDs to TQDs is a function of the temperature. Furthermore, there is an interplay between the temperature and excitation, as can be seen by the different temperature dependent " $W$ " profiles obtained by changing the excitation intensity for the same bilayer QD sample.

\section{Conclusions}

In summary, we have studied the optical properties of a coupled bilayer InAs/GaAs QD structure. PL measurements with different excitation intensities and wavelengths at low temperature show that the carrier transfer between dot pairs is strongly dependent on the carrier injection from the excitation laser. The temperature-dependent PL-in consideration of the emission wavelength, peak position, FWHM, and intensity ratio-indicate that the carrier transfer inside this bilayer QD structure depends on temperature and excitation intensity. This result demonstrates that there is a clear interplay between temperature and injection on the vertical transfer of carriers between the SQD layer and the TQD layer.

Acknowledgments: The authors acknowledge the financial support by the "Hebei Province 100-Talents Program" (Grant \# E2013100013) and Natural Science Foundation of Hebei Province (Grant \# A2012201013) of China. 
This research is also supported by the National Science Foundation of the U.S. (Grant \# DMR-1309989 and EPSCoR Grant \# OIA-1457888).

Author Contributions: Shufang Wang, Guangsheng Fu and Gregory J. Salamo conceived and designed the experiments; Baolai Liang and Yuriy I. Mazur performed the MBE growth of the samples. Yao Liu, Ying Wang and Qinglin Guo performed the optical experiments and analyzed the data; Yao Liu, Baolai Liang, Yuriy I. Mazur and Morgan E. Ware wrote the paper.

Conflicts of Interest: The authors declare no conflict of interest.

\section{References}

1. Bimberg, D.; Grundmann, M.; Ledentsov, N.N. Quantum Dot Heterostructures; Wiley: New York, NY, USA, 1998.

2. Yuan, Z.L.; Kardynal, B.E.; Stevenson, R.M.; Shields, A.J.; Lobo, C.J.; Cooper, K.; Beattie, N.S.; Ritchie, D.A.; Pepper, M. Electrically driven single-photon source. Science 2002, 295, 102-105. [CrossRef] [PubMed]

3. Leonard, D.; Pond, K.; Petroff, P.M. Critical layer thickness for self-assembled InAs islands on GaAs. Phys. Rev. B 1994, 50, 11687-11692. [CrossRef]

4. Nishibayashi, K.; Kawazoe, T.; Ohtsu, M.; Akahane, K.; Yamamoto, N. Observation of interdot energy transfer between InAs quantum dots. Appl. Phys. Lett. 2008, 93, 042101. [CrossRef]

5. Fry, P.W.; Itskevich, I.E.; Mowbray, D.J.; Skolnick, M.S.; Finley, J.J.; Barker, J.A.; O’Reilly, E.P.; Wilson, L.R.; Larkin, I.A.; Maksym, P.A.; et al. Inverted electron-hole alignment in InAs-GaAs self-assembled quantum dots. Phys. Rev. Lett. 2000, 84, 733-736. [CrossRef] [PubMed]

6. Bhattacharya, P.; Ghosh, S.; Stiff-Roberts, A.D. Quantum dot opto-eletronic devices. Annu. Rev. Mater. Res. 2004, 34, 1-40. [CrossRef]

7. Huffaker, D.L.; Park, G.; Zou, Z.; Shchekin, O.B.; Deppe, D.G. $1.3 \mu \mathrm{m}$ room-temperature GaAs-based quantum-dot laser. Appl. Phys. Lett. 1998, 73, 2564-2566. [CrossRef]

8. Bailey, C.G.; Forbes, D.V.; Raffaelle, R.P.; Hubbard, S.M. Near 1 V open circuit voltage InAs/GaAs quantum dot solar cells. Appl. Phys. Lett. 2011, 98, 163105. [CrossRef]

9. Horiguchi, N.; Futatsugi, T.; Nakata, Y.; Yokoyama, N.; Mankad, T.; Petroff, P.M. Quantum dot infrared photodetector using modulation doped InAs self-assembled quantum dots. J. Appl. Phys. 1999, 38, 2559-2561. [CrossRef]

10. Liu, H.Y.; Wang, T.; Jiang, Q.; Hogg, R.; Tutu, F.; Pozzi, F.; Seeds, A. Long-wavelength InAs/GaAs quantum-dot laser diode monolithically grown on Ge substrate. Nat. Photonics 2011, 5, 416-419. [CrossRef]

11. Li, X.Q.; Wu, Y.W.; Steel, D.; Gammon, D.; Stievater, T.H.; Katzer, D.S.; Park, D.; Piermarocchi, C.; Sham, L.J. An all-optical quantum gate in a semiconductor quantum dot. Science 2003, 301, 809-811. [CrossRef] [PubMed]

12. Wu, J.; Hu, X.; Lee, J.; Kim, E.S.; Wang, Z.M. Epitaxially self-assembled quantum dot pairs. Adv. Opt. Mater. 2013, 1, 201-214. [CrossRef]

13. Fino, N.R.; Camacho, A.S.; Ramírez, H.Y. Coupling effects on photoluminescence of exciton states in asymmetric quantum dot molecules. Nanoscale Res. Lett. 2014, 9, 297. [CrossRef] [PubMed]

14. Ji, H.M.; Liang, B.L.; Simmonds, P.J.; Juang, B.C.; Yang, T.; Young, R.J.; Huffaker, D.L. Hybrid type-I InAs/GaAs and type-II GaSb/GaAs quantum dot structure with enhanced photoluminescence. Appl. Phys. Lett. 2015, 106, 103104. [CrossRef]

15. Chakrabarti, S.; Halder, N.; Sengupta, S.; Ghosh, S.; Mishima, T.D.; Stanley, C.R. Vertical ordering and electronic coupling in bilayer nanoscale InAs/GaAs quantum dots separated by a thin spacer layer. Nanotechnology 2008, 19, 505704. [CrossRef] [PubMed]

16. Mazur, Y.I.; Wang, Z.M.; Tarasov, G.G.; Kunets, V.P.; Salamo, G.J.; Zhuchenko, Z.Y.; Kissel, H. Tailoring of high-temperature photoluminescence in InAs/GaAs bilayer quantum dot structures. J. Appl. Phys. 2005, 98, 053515. [CrossRef]

17. Bayer, M.; Hawrylak, P.; Hinzer, K.; Fafard, S.; Korkusinski, M.; Wasilewski, Z.R.; Stern, O.; Forchel, A. Coupling and entangling of quantum states in quantum dot molecules. Science 2001, 291, 451-453. [CrossRef] [PubMed] 
18. Tarasov, G.G.; Zhuchenko, Z.Y.; Lisitsa, M.P.; Mazur, Y.I.; Wang, Z.M.; Salamo, G.J.; Warming, T.; Bimberg, D.; Kissel, H. Optical detection of asymmetric quantum-dot molecules in double-layer InAs/GaAs structures. Semiconductors 2006, 40, 79-83. [CrossRef]

19. Liu, Y.; Liang, B.L.; Guo, Q.L.; Wang, S.F.; Fu, G.S.; Fu, N.; Wang, Z.M.; Mazur, Y.I.; Salamo, G.J. Electronic coupling in nanoscale InAs/GaAs quantum dot pairs separated by a thin $\mathrm{Ga}(\mathrm{Al}) \mathrm{As}$ spacer. Nanoscale Res. Lett. 2015, 10, 271. [CrossRef] [PubMed]

20. Levi, D.H.; Wake, D.R.; Klein, M.V.; Kumar, S.; Morkoc, H. Density dependence of nonresonant tunneling $\ln$ asymmetric coupled quantum wells. Phys. Rev. B 1992, 45, 4274-4279. [CrossRef]

21. Mazur, Y.I.; Wang, Z.M.; Tarasov, G.G.; Xiao, M.; Salamo, G.J.; Tomm, J.W.; Talalaev, V.; Kissel, H. Interdot carrier transfer in asymmetric bilayer InAs/GaAs quantum dot structures. Appl. Phys. Lett. 2005, 86, 063102. [CrossRef]

22. Jin, S.R.; Zheng, Y.L.; Li, A.Z. Characterization of photoluminescence intensity and efficiency of free excitons in semiconductor quantum well structures. J. Appl. Phys. 1997, 82, 3870-3873. [CrossRef]

23. Martini, S.; Quivy, A.A.; Tabata, A.; Leite, J.R. Influence of the temperature and excitation power on the optical properties of InGaAs/GaAs quantum wells grown on vicinal GaAs(001) surfaces. J. Appl. Phys. 2001, 90, 2280-2289. [CrossRef]

24. Mazur, Y.I.; Dorogan, V.G.; Guzun, D.; Marega, E., Jr.; Salamo, G.J.; Tarasov, G.G.; Govorov, A.O.; Vasa, P.; Lienau, C. Measurement of coherent tunneling between InGaAs quantum wells and InAs quantum dots using photoluminescence spectroscopy. Phys. Rev. B 2010, 82, 155413. [CrossRef]

25. Shih, C.I.; Lin, C.H.; Lin, S.C.; Lin, T.C.; Sun, K.W.; Voskoboynikov, O.; Lee, C.P.; Suen, Y.W. Effects of crossed states on photoluminescence excitation spectroscopy of InAs quantum dots. Nanoscale Res. Lett. 2011, 6, 409. [CrossRef] [PubMed]

26. Mazur, Y.I.; Lopes-Oliveira, V.; Souza, L.D.; Lopez-Richard, V.; Teodoro, M.D.; Dorogan, V.G.; Benamara, M.; $\mathrm{Wu}$, J.; Tarasov, G.G.; Marega, J.E.; et al. Carrier transfer in vertically stacked quantum ring-quantum dot chains. J. Appl. Phys. 2015, 117, 154307. [CrossRef]

27. Chen, J.X.; Markus, A.; Fiore, A.; Oesterle, U.; Stanley, R.P.; Carlin, J.F.; Houdré, R.; Ilegems, M.; Lazzarini, L.; Nasi, L.; et al. Tuning InAs/GaAs quantum dot properties under Stranski-Krastanov growth mode for $1.3 \mu \mathrm{m}$ applications. J. Appl. Phys. 2002, 91, 6710-6716. [CrossRef]

28. Sanguinetti, S.; Henini, M.; Grassi Alessi, M.; Capizzi, M.; Frigeri, P.; Franchi, S. Carrier thermal escape and retrapping in self-assembled quantum dots. Phys. Rev. B 1999, 60, 8276-8283. [CrossRef] 